\title{
A Case of ALERD Masquerading as Paracetamol Poisoning
}

\section{Siddarameshwar S Kalyanshettar, Shankargouda V Patil, Prakash VJ, Asif Mahaldar and Prashant Teggi}

Department of Paediatrics, Sri BM Patil Medical College Hospital and Research Centre, BLDE (Deemed to be university), Vijayapura, Karnataka, India

\section{Correspondence:}

Siddarameshwar S Kalyanshettar

Department of Paediatrics

Sri BM Patil Medical College Hospital and Research Centre,

BLDE (Deemed to be university).

Vijayapura, Karnataka, India

Email: s.kalyanshettar@bldedu.ac.in

DOI: $10.3126 /$ jnps.v40i3.29728

Submitted on: $2020-06-27$

Accepted on: 2020-10-04

Acknowledgements: None

Funding: Nil

Conflict of Interest: None declared

Permission from IRB: Yes

To cite this article: Kalyanshettar SS, Patil SV, Prakash VJ, Mahaldar A, Teggi P. A Case of ALERD Masquerading as Paracetamol Poisoning. J Nepal Paediatr Soc. 2020;40(3):261-4.

\begin{abstract}
ALERD (Acute leukoencephalopathy with restricted diffusion) is a clinico - radiological diagnosis. Many novel causes of acute encephalopathy are emerging in children. MRI plays an important role in the diagnosis and management of such children. Many infectious and non-infectious conditions and poisonings are known to precipitate acute leukoencephalopathy. A commonly used drug like paracetamol ingested in large quantities is known to cause varied effects. We describe one such child who presented to us with paracetamol poisoning and developed acute leukoencephalopathy. He was later found to have restricted diffusion on magnetic resonance imaging.
\end{abstract}

Key words: ALERD; Paracetamol poisoning; Biphasic seizures

\section{1.}

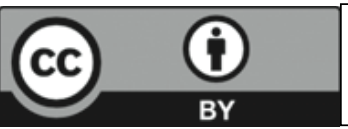

This work is licensed under creative common attribution 3.0 license 


\section{INTRODUCTION}

Acute encephalopathy in children can have varied etiologies like infections and non-infectious causes including poisonings. Acute leukoencephalopathy with restricted diffusion is a clinico - radiological diagnosis, which is being increasingly reported in recent years. MRI plays an important role in the diagnosis and management of such children. We describe one such child who presented to us with paracetamol poisoning and developed acute leukoencephalopathy subsequently and was found to have restricted diffusion on magnetic resonance imaging.

\section{CASE REPORT}

A three year old boy weighing $10 \mathrm{~kg}$ presented with alleged history of consumption of $40 \mathrm{ml}$ of syrup paracetamol $250 \mathrm{mg} / 5 \mathrm{ml}(200 \mathrm{mg} / \mathrm{kg}$ of paracetamol). Stomach wash was given by a local paediatrician and referred to our hospital for further care. Child was hemodynamically stable at admission and evaluated for paracetamol poisoning and started with $\mathrm{N}$-acetyl cysteine at dose of 300 $\mathrm{mg} / \mathrm{kg}$ in divided doses. CBC at admission was normal with mild elevation of liver enzymes. Serum paracetamol levels were within normal limits. Serum paracetamol was 5.9 micro gram $/ \mathrm{ml}$. Toxicologic analysis of gastric contents detected paracetamol.

On second day of admission, child developed sudden onset respiratory distress and required ventilator support in view of poor respiratory efforts \& dipping oxygen saturation. On the same day, child developed convulsions involving left side of body. The blood sugar and calcium levels were normal and anticonvulsants were initiated as per PICU protocol. Child's sensorium worsened gradually and required ventilator support for further two days. On fourth day antibiotics were upgraded in view of persistent fever and worsening sensorium. Lumbar puncture was done after ruling out increased intracranial tension. However CSF analysis was normal.

On fifth day of admission patient could be weaned off ventilator and second anticonvulsant oxcarbazepine was started in view of recurrent convulsions. MRI brain was done. MRI brain revealed symmetrical hyper intensity showing restricted diffusion on DWI with corresponding low signals on ADC in bilateral temporo-parietooccipital, right frontal and splenium of corpus callosum, without evidence of blooming on GRE, features suggestive of acute leukoencephalopathy with restricted diffusion. (Figure 1)

Child showed some improvement of sensorium but continued to have depressed deep tendon reflexes $\&$ hypotonia. Child was not oriented. Paediatric neurologist consultation was sought and started on methylprednisolone ( $25 \mathrm{mg} / \mathrm{kg} /$ day) for seven days, cyclosporin (2 $\mathrm{mg} / \mathrm{kg} /$ day) and dextromethorphan (5 $\mathrm{mg} /$ day). Nasogastric feeds were started on sixth day and gradually increased. Sensorium improved further and child tolerated full oral feeds from tenth day. Child was discharged on fifteenth day with oral cyclosporin, dextromethorphan and oxcarbazepine. At discharge child was conscious and hearing normally but had cortical blindness.

\section{DISCUSSION}

Acute encephalopathy refers to acute insult to the brain and presents with seizures, impaired consciousness. ${ }^{1}$ Common causes during childhood include febrile encephalopathy, systemic disorders, hypoxic-ischemic encephalopathy, toxins, poisonings and stroke. ${ }^{2,3}$

ALERD (Acute leukoencephalopathy with restricted diffusion) is a clinico-radiological syndrome which has a low mortality rate but has a higher incidence of neurologic sequelae. Clinical

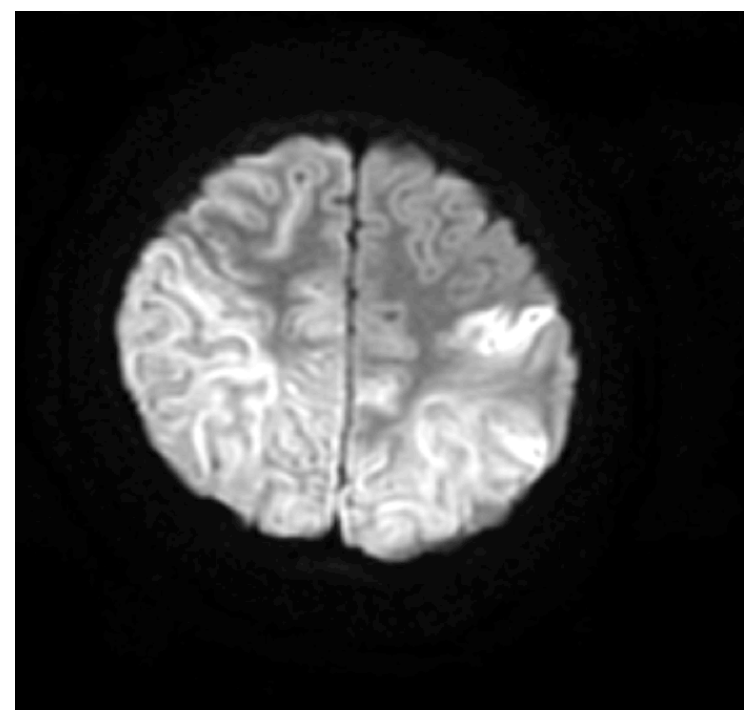

Figure 1. Showing MRI image of encephalopathy with restricted diffusion 
features include fever, altered sensorium and prolonged seizures. ${ }^{4}$ Pathogenesis of the condition is hypercytokinemia as seen in many patients in the form of elevated IL-6, IL-8, IL-10 and tumour necrosis factor in the serum, and IL-4, IL-6 and IL-8 in the cerebrospinal fluid (CSF). Excitotoxic injury with delayed (or apoptotic) neuronal death is hypothesised as a possible mechanism..$^{5}$ There are reports of cases of ALERD associated with hemophagocytic syndrome, thereby suggesting the role of hypercytokinemia as a pathogenesis of ALERD. ${ }^{5}$ Unlike autoimmune encephalitis, it is not thought to be an immune-mediated disorder. The pathophysiological mechanism of ALERD does not require direct infection of the central nervous system. Certain genetic predisposition factors for ALERD include certain genotype of carnitine palmitoyl transferase-II, SCN1A, SCN2A, ADORA2A and TLR3.5,6

Two types of ALERD are described - diffuse and central. Diffuse ALERD is a severe phenotype with rapid and severe deterioration of consciousness. Coma is observed within 24 hours after onset of disease. The outcome is poor and can be associated with mortality. Sequelae include cognitive impairment, epilepsy and behavioural problems. Central ALERD is a relatively mild phenotype of acute encephalopathy characterised by biphasic clinical course. Onset is often marked by prolonged seizure with fever followed by impaired consciousness. Clustered seizures, signs of frontal lobe dysfunction and worsening of consciousness become apparent at three to four days after onset. Investigations reveal increased liver enzymes, hyperglycemia and metabolic acidosis. CSF examination is usually normal. CSF levels of inflammatory cytokines, such as IL-6 and tumour necrosis factor are markedly elevated. ${ }^{1}$ Treatment includes intravenous pulse steroids and or intravenous immunoglobin. ${ }^{1}$ Other possible treatment include combination therapy of $\mathrm{N}$ methyl-D-aspartate receptor antagonist, dextromethorphan and apoptosis inhibitor, cyclosporine A etc. ${ }^{7}$ Similarly, other agents reported as potentially effective for ALERD include fosphenytoin, L-carnitine and vitamins, and erythropoietin. ${ }^{8}$ Overall mortality is $5 \%-10 \%$ and incidence of postencephalopathic epilepsy is $23 \%{ }^{4}$ Other long-term sequelae are cognitive impairment, hyperactivity with speech delay, spasticity, dyskinesias and hemiparesis.

Our patient continued to have cortical blindness even six months after the episode and no recurrence of seizures. After extensive literature search, we could not find any case of ALERD associated with paracetamol poisoning. This could be the first such case.

\section{CONCLUSIONS}

ALERD can occur secondary to infections and poisonings. Paracetamol poisoning may rarely present with acute leukoencephalopathy with ALERD. Sudden deterioration of neurological status and seizures in any child with normal CSF findings should raise suspicion of such conditions. MRI brain helps in early diagnosis. Early treatment with pulse steroids with anti-convulsants is the treatment of choice.

\section{REFERENCES}

1. Okumura A, Kidokoro H, Tsuji T, Suzuki M, Kubota T, Kato T, et al. Differences of clinical manifestations according to the patterns of brain lesions in acute encephalopathy with reduced diffusion in the bilateral hemispheres. AJNR Am J Neuroradiol. 2009;30:825-30., DOI:10.3174/ajnr.A1431

2. Mizuguchi M, Abe J, Mikkaichi K, Noma S, Yoshida K, Yamanaka T, et al. Acute necrotising encephalopathy of childhood: A new syndrome presenting with multifocal, symmetric brain lesions. J Neurol Neurosurg Psychiatry. 1995;58(5):555-61. DOI: 10.1136/jnnp.58.5.555.

3. Tada H, Takanashi J, Barkovich AJ, Oba H, Maeda M, Tsukahara H, et al. Clinically mild encephalitis/ encephalopathy with a reversible splenial lesion. Neurology. 2004;63:1854-8. DOI:10.1212/01.wnl. 0000144274.12174.cb

4. Singh V, Tomar V, Kumar A, Phadke RV. Acute leucoencephalopathy with restriction of diffusion - A case report. Eastern J Med. 2012;17:149-52. 
5. Saitoh M, Shinohara M, Hoshino H, Kubota M, Amemiya K, Takanashi JL, et al. Mutations of the SCN1A gene in acute encephalopathy. Epilepsia. 2012;53:558-64. DOI:10.1111/j.1528-1167.2011.03402.x.

6. Saitoh M, Shinohara M, Ishii A, Ihara Y, Hirose S, Shiomi M, et al. Clinical and genetic features of acute encephalopathy in children taking theophylline. Brain Dev. 2015;37:463-70. DOI:10.1016/j.braindev.2014.07.010.

7. Matsuo M, Maeda T, Ono N, Sugihara S, Kobayashi I, Koga D, et al. Efficacy of dextromethorphan and cyclosporine a for acute encephalopathy. Pediatr Neurol. 2013;48:200-5. DOI:10.1016/j.pediatrneurol.2012.11.003

8. Nakazawa M, Akasaka M, Hasegawa T, Suzuki T, Shima T, Takanashi J, et al. Efficacy and safety of fosphenytoin for acute encephalopathy in children. Brain Dev. 2015;37:418-22. DOI: 10.1016/j.braindev.2014.06.009 